\title{
Multiple Adult Xanthogranuloma: A Rare Case Report with \\ Characteristics
}

\author{
Xin $\operatorname{Tian}^{1,2^{\ddagger}}$, Suling $\mathrm{He}^{1,2^{\ddagger}}$, Runxiang $\mathrm{Li}^{1,2^{*}}$, Jianqin Wang ${ }^{1,2^{*}}$ \\ ${ }^{1}$ Institute of Dermatology, Guangzhou Medical University, Guangzhou 510095, P.R. China \\ ${ }^{2}$ Department of Dermatology, Guangzhou Institute of Dermatology, Guangzhou 510095, P.R. China
}

Corresponding Author(s): Runxiang Li and Jianqin Wang

Address: Department of Dermatology, Guangzhou Institute of Dermatology, 56 Hengfu Road, Guangzhou 510o95, P.R. China.

${ }^{\ddagger}$ These two authors contributed equally to this work.

Received date: 19 February 2021; Accepted date: 05 April 2021; Published date: 12 April 2021

Citation: Tian X, He S, Li R, Wang J. Multiple Adult Xanthogranuloma: A Rare Case Report with Characteristics. Asp Biomed Clin Case Rep. 2021 Apr 12;4(2):88-92.

Copyright (C) 2021 Tian X, He S, Li R, Wang J. This is an open-access article distributed under the Creative Commons Attribution License, which permits unrestricted use, distribution, and reproduction in any medium provided the original work is properly cited.

\begin{abstract}
Multiple adult xanthogranuloma (MAXG) is an uncommon adult form of xanthogranuloma. The clinical manifestation of MAXG is relatively rare, but it has characteristic histopathological and immunohistochemical features. MAXG is mainly associated with cutaneous damage, and extracutaneous manifestations such as cervical spine, intracardiac, and periocular lesions have rarely been reported. In patients with systemic association, clinicians should pay attention to the possibility of hematological malignancies. We report the case of a 17-year-old man with multiple lesions on the head, face, neck, arms and trunk, along with the clinical manifestations and pathological features.
\end{abstract}

\section{Keywords}

Multiple Adult Xanthogranuloma, Systemic Association, Touton Giant Cell, Xanthogranuloma, Case Report

\section{Introduction}

Xanthogranuloma (XG) is a relatively common form of non-Langerhans cell histiocytosis that mostly involves to the skin and/or mucosa. The etiology and pathogenesis of XG remain uncertain, and it can be classified into juvenile xanthogranuloma (JXG) or adult xanthogranuloma (AXG) according to the age of onset. The most frequent adult manifestation of $X G$ is a solitary nodule, and multiple adult xanthogranuloma (MAXG) can be defined by the presence of more than five lesions in patients over 14 years of age [1]. The appearance of multiple lesions is unusual in adults. Here, we report the case of a 17-year-old man diagnosed with MAXG, along with the clinical manifestations and pathological characteristics.

\section{Case Report}

A 17-year-old male patient with a 6-month history of multiple painless red to yellowish-brown papules and nodules was consulted at Guangzhou Institute of Dermatology in July 2017. The patient was healthy before, and he had no remarkable personal history, family history, infection history or drug history, nor did he smoke or use alcohol. No obvious positive symptoms were observed. Physical examinations showed multiple subcutaneous papules and nodules on the head, face, neck, arms and the upper part of the trunk, colored from red to yellowish-brown (Fig-1). 
Citation: Tian X, He S, Li R, Wang J. Multiple Adult Xanthogranuloma: A Rare Case Report with Characteristics. Asp Biomed Clin Case Rep. 2021 Apr 12;4(2):88-92.

The distribution was asymmetric. The lesions had smooth surfaces and were soft on palpation, with diameters from 5 to $20 \mathrm{~mm}$ and no tenderness. Laboratory tests revealed no abnormalities in routine blood examination parameters, liver function, renal function or triglyceride levels. The initial considerations were eruptive xanthoma, sarcoidosis, cutaneous lymphoma or cutaneous metastatic tumor. To clarify the diagnosis, excisional biopsy was performed on a lesion.

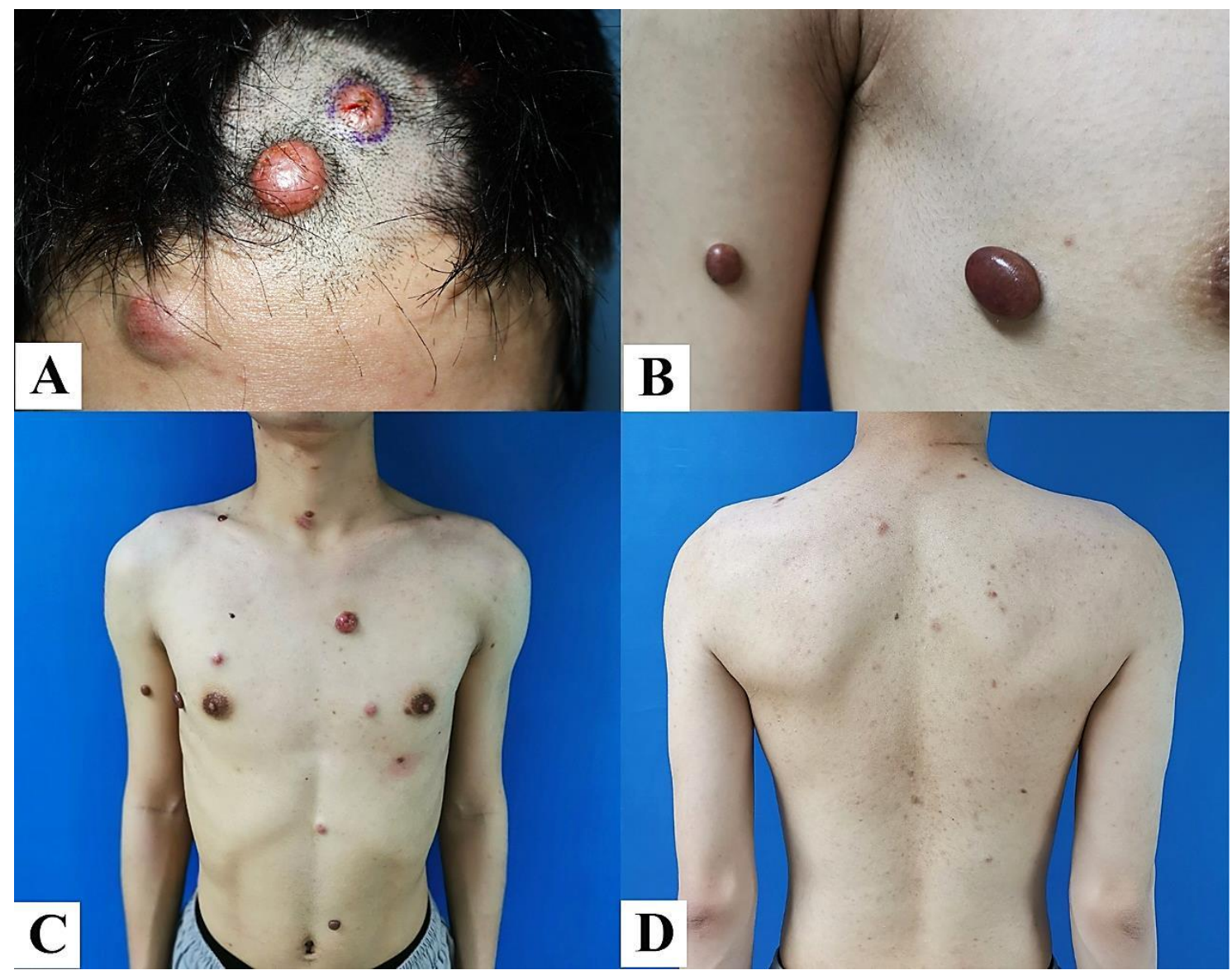

Fig-1:

Clinical features of multiple adult xanthogranuloma. A, B, C and D, Multiple subcutaneous papules and nodules on the head, face, neck, arms and the upper part of the trunk that are asymmetric and colored from red to yellowish-brown.

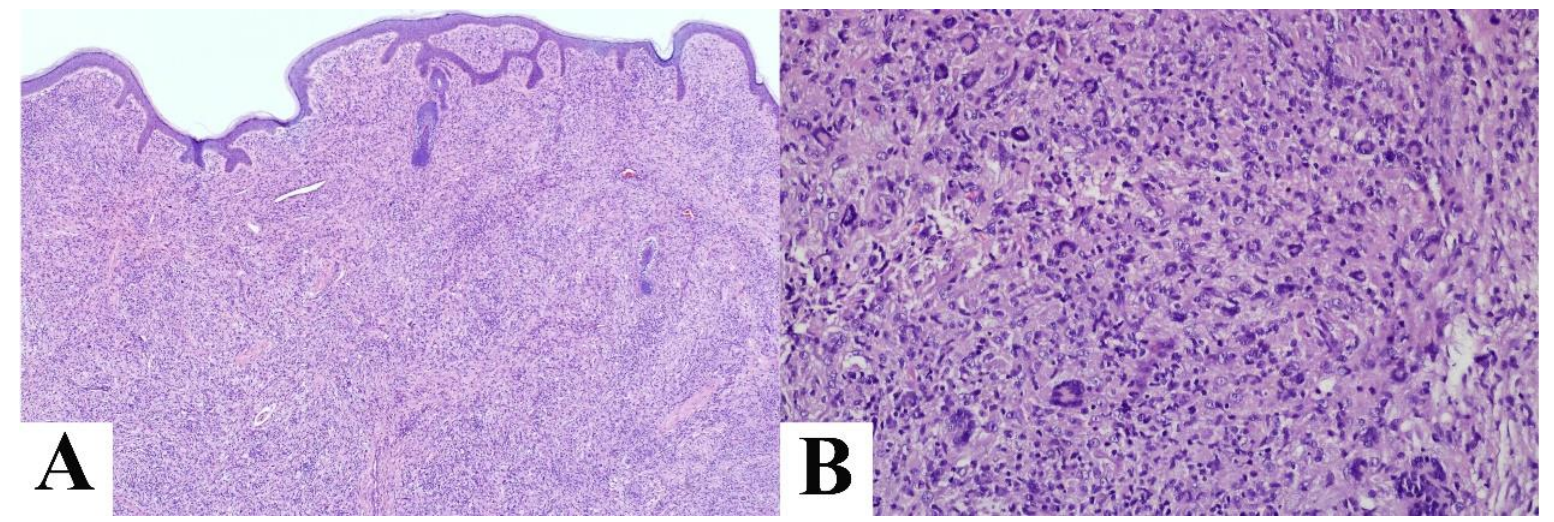

\section{Fig-2:}

A, The epidermis was thinner with a disappearing epidermal process and mixed proliferation of histiocytes in the dermis (H\&E stain $\times 40$ ). B, Infiltration of histiocytes with foamy cytoplasm in the dermis and subcutaneous tissues, accompanied by multinucleated Touton giant cells (H\&E stain $\times 200$ ). 
Histopathological examination revealed a predominant infiltration of histiocytes with foamy cytoplasm in the dermis and subcutaneous tissues, characterized by Touton giant cells, occasional lymphocytes and rare eosinophils (Fig-2). Immunohistochemical staining showed diffuse CD68positive cells, scattered individual CD1a-positive cells, and S10o-negative cells (Fig-3). The Ki67 proliferation index was approximately $3 \%$. The diagnosis of MAXG was made on the basis of the clinical manifestation and the histopathological and immunohistochemical findings. From July 2017 to April 2019, the patient had been treated with intradermal injection of triamcinolone acetonide injection combined with local surgical resection intermittently. After treatment, the nodules were smaller and soften than before. Regrettably, the patient did not continue to visit for personal reasons. During telephone follow-up, there was no significant change in the skin lesions, with no new papules and nodules, and no recurrence in the original resection site.

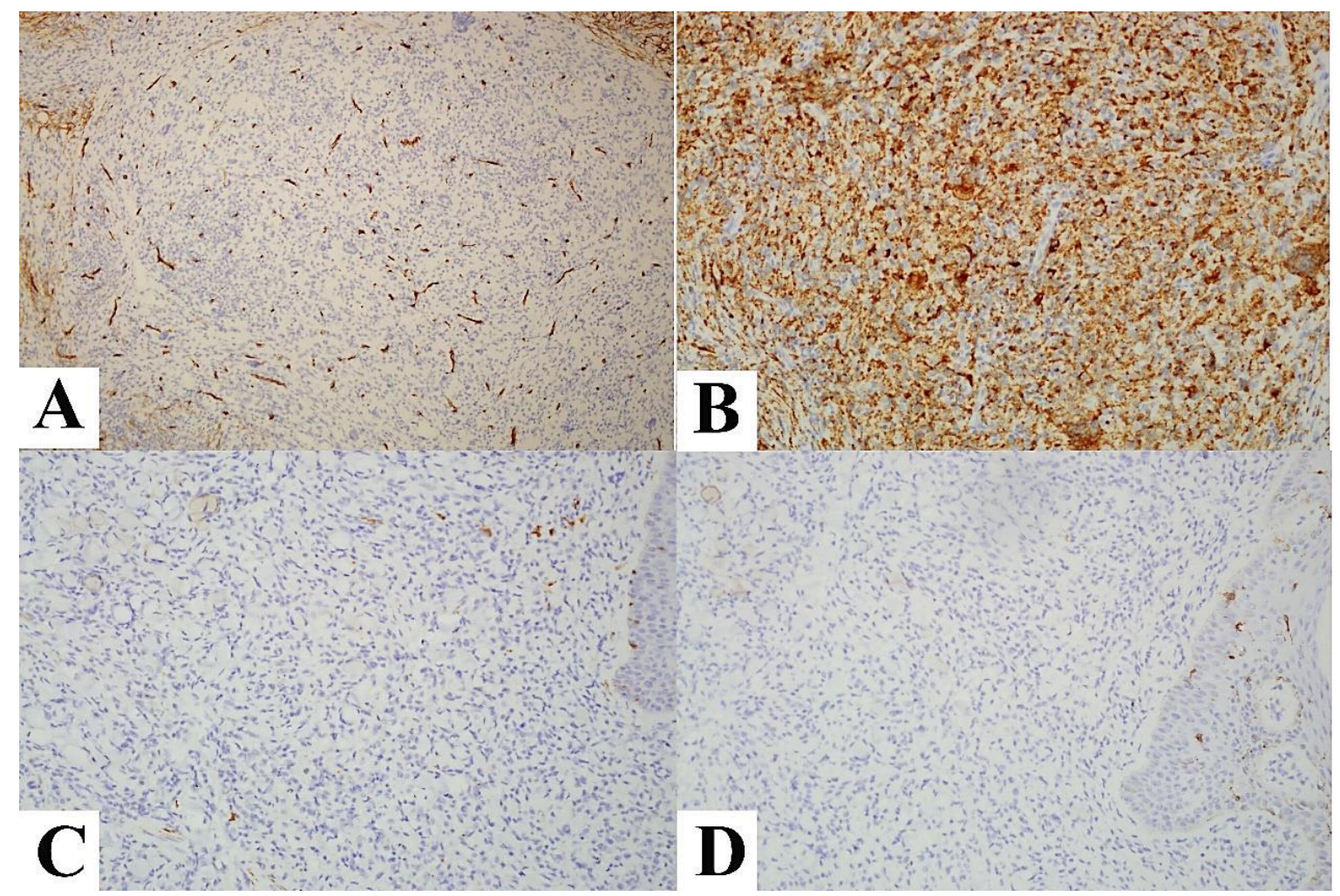

Fig-3:

Immunohistochemistry positive for $C D 68$ (B) and negative for $C D 34(A), C D 1 a(C)$ as well as S1oo protein $(D)$; $(A-D$, $\times 200$ ).

\section{Discussion}

XG mainly occurs in infancy and early childhood, and adults can also be affected. The lesions of XG can manifest as single or multiple reddish to yellowishbrown papules and nodules on the head, neck, limbs, or trunk. In addition to cutaneous damage, JXG may be accompanied by other extracutaneous XG lesions of the eyes, lungs, liver, testes, central nervous system or kidneys. JXG is more likely to be associated with systemic manifestations, such as neurofibroma, morphea, organoid nevus and lymph node enlargement [1]. Children with neurofibromatosis type
1 and XG have been estimated to have a 20- to 32-fold higher risk for chronic myelocytic leukemia (CML) [2].

AXG is mainly associated with the skin, and extracutaneous manifestations such as cervical spine, intracardiac, and periocular lesions have been rarely reported [3]. In patients with multiple lesions, clinicians should pay attention to the possibility of hematological malignancies. Relevant diseases reported in the literature include primary thrombocytopenia, chronic lymphoblastic leukemia, large B-cell lymphoma, and monoclonal gammopathy 
[4]. A retrospective literature research showed that AXG accounted for approximately $15 \%$ of XG [5]; the ratio of solitary lesions by sex was $0.9: 1$ for men:women and that of multiple lesions was 1.6:1 for men:women [6]. Compared with JXG, the proportion of spontaneous regression was lower. Some scholars believe that XG may be a reactive granuloma response of histiocytes to infection, trauma, physical stimulation, malignant tumors or unknown stimuli. However, except for the inflammatory environment that may lead to the activation of histiocytes, the mechanism is still unclear.

Lesions in AXG can be single or multiple, usually larger than those of JXG. The initial appearance is a round or oval reddish papule of rice grain size with a clear boundary. Gradually, the lesion may turn yellow or brown and slowly enlarge with a diameter of 1-20 $\mathrm{mm}$, and AXG lesions mostly involve the face, limbs, and trunk. The pathologies of AXG and JXG are essentially consistent [7]. In the early stage, histopathological examination reveals simple morphological histiocyte infiltration. As the disease progresses, the histiocytes mature and become diverse. Foamy histiocytes, spindle monocytes, foreign body giant cells and Touton giant cells can be observed under microscopic observation [3]. The cytoplasm of Touton giant cells is foamy, and the nucleus is shaped like a circular flower ring, which indicates the diagnostic character [8]. A variety of inflammatory cells, such as lymphocytes, neutrophils, plasma cells or eosinophils, proliferate between histiocytes. Immunohistochemical examination shows positive expression of CD68 and vimentin and negative expression of CD1a and S10o. Moreover, staining for factor XIIIa is positive, which helps in the diagnosis of XG [6]. To summarize, the clinical manifestations, pathology and immunohistochemistry of this patient were in accordance with the diagnosis of MAXG.

MAXG needs to be differentiated from eruptive xanthoma, xanthoma disseminatum, progressive nodular histiocytoma, generalized eruptive histiocytoma and other relevant diseases. Eruptive xanthoma is expressed as needle-sized yellow or orange papules surrounded by inflammatory red halos that usually occur on the buttocks, hands and feet.
Most importantly, it is often accompanied by hypertriglyceridemia. Xanthoma disseminatum presents as yellow-brown papules or nodules that are widely distributed in flexional and interfacial positions. Moreover, dyspnea can be caused if the respiratory tract mucous membrane is invaded. When the hypothalamus-pituitary region is involved, diabetes insipidus and occult diabetes mellitus can exist. Progressive nodular histiocytosis is characterized by reddish-brown and yellowish-brown superficial papules or deep fibrous nodules. Some severe cases manifest as typical leonine facies. Histologically, spindled cell xanthogranuloma indicates the diagnosis. Generalized eruptive histiocytoma can be distinguished by centripetal reddish-brown papules with a symmetrical distribution in the proximal part of the trunk and limbs, which is hardly involved with mucosa. There is no Touton giant cell infiltration in histological biopsy.

AXG usually resolves spontaneously but at a lower rate than JXG. If the single lesion does not subside, surgical resection can be considered. Glucocorticoids or combined chemotherapy may be systematically applied if the lesions are multiple or complicated with systemic damage.

\section{Conflicts of Interest}

All authors have read and approved the final version of the manuscript. The authors have no conflicts of interest to declare.

\section{Acknowledgments}

We thank our clinical colleagues, and the patient who contributed the sample used in this study. This work was supported by the Health Science and Technology Project of Guangzhou (20191A011070).

\section{References}

[1] Chang SE, Cho S, Choi JC, Choi JH, Sung KJ, Moon KC, Koh JK. Clinicohistopathologic comparison of adult type and juvenile type xanthogranulomas in Korea. J Dermatol. 2001 Aug;28(8):413-18. [PMID: 11560157]

[2] Zvulunov A, Barak Y, Metzker A. Juvenile xanthogranuloma, neurofibromatosis, and juvenile chronic myelogenous leukemia. World statistical analysis. Arch Dermatol. 1995 Aug;131(8):904-o8. 
[PMID: 7632061]

[3] Chisolm SS, Schulman JM, Fox LP. Adult Xanthogranuloma, Reticulohistiocytosis, and RosaiDorfman Disease. Dermatol Clin. 2015 Jul;33(3):46572. [PMID: 26143426]

[4] Shoo BA, Shinkai K, McCalmont TH, Fox LP. Xanthogranulomas associated with hematologic malignancy in adulthood. J Am Acad Dermatol. 2008 Sep;59(3):488-93. [PMID: 18538449]

[5] Shin SJ, Scamman W, Gopalan A, Rosen PP. Mammary presentation of adult-type "juvenile" xanthogranuloma. Am J Surg Pathol. 2005 Jun;29(6):827-31. [PMID: 15897751]
[6] Saad N, Skowron F, Dalle S, Forestier JY, Balme B, Thomas L. Multiple adult xanthogranuloma: case report and literature review. Dermatology. 2006;212(1):73-76. [PMID: 16319479]

[7] Ederle A, Kim KH, Gardner JM. Eruptive xanthogranuloma in a healthy adult male. J Cutan Pathol. 2017 Apr;44(4):385-87. [PMID: 28000234]

[8] Tan LC, Tan KB, Aw CW. Unusual presentation of adult xanthogranuloma: a case report. Singapore Med J. 2014 Feb;55(2):e25-27. Erratum in: Singapore Med J. 2014 Mar;55(3):173. Tan, Kong Bing [added]. [PMID: 24154556] 